\title{
Macroscopic model for head-on binary droplet collisions in a gaseous medium $^{\mathrm{a})}$
}

\author{
Jie $\mathrm{Li}^{1}$ \\ Department of Engineering, University of Cambridge
}

(Dated: 14 September 2016)

\begin{abstract}
In this letter, coalescence-bouncing transitions of head-on binary droplet collisions are predicted by a novel macroscopic model based entirely on fundamental laws of physics. By making use of the lubrication theory of Zhang and Law [Phys. Fluids 23, 042102 (2011)], we have modified the Navier-Stokes equations to accurately account for the rarefied nature of the interdroplet gas film. Through the disjoint pressure model, we have incorporated the intermolecular Van der Waals forces. Our model does not use any adjustable (empirical) parameters. It therefore encompasses an extreme range of length scales (more than 5 orders of magnitude): from those of the external flow in excess of the droplet size (a few hundred $\mu \mathrm{m}$ ) to the effective range of the Van der Waals force around $10 \mathrm{~nm}$. A state of the art moving adaptive mesh method, capable of resolving all the relevant length scales, has been employed. Our numerical simulations are able to capture the coalescencebouncing and bouncing-coalescence transitions that are observed as the collision intensity increases. The predicted transition Weber numbers for tetradecane and water droplet collisions at different pressures show good agreement with published experimental values. Our study also sheds new light on the roles of gas density, droplet size and mean free path in the rupture of the gas film.
\end{abstract}

Keywords: Thin film, lubrication theory, rarefiled gas dynamics, Van der Waals' forces, disjoint pressure, Navier-Stokes equations, Boltzmann equations, moving adaptive mesh method

Drop impact on a solid surface ${ }^{1-4}$ and droplet collision $^{5-8}$ are fascinating multi-phase flow problems. Not only are these subjects of importance in understanding many natural and technological processes, they are also interesting from the fundamental point of view. They have been very active areas of research for the last twenty years ${ }^{9-18}$. Currently, one of the greatest puzzles in multiphase flows is: why does the surrounding air play a dramatic role in determining the outcome of drop impact and droplet collision? In fact, when the ambient air pressure is reduced, two amazing phenomena have been observed experimentally: (1) the bouncing regime of two colliding tetradecane droplets may disappear ${ }^{19} ;(2)$ the splashing of an impacting drop can be suppressed ${ }^{20}$. The outcomes of these beautiful experiments are easy to comprehend, yet the underlying suppression mechanism is unknown. It is not obvious which physical property of the air is important. We note that the air dynamic viscosity and the surface tension coefficient do not vary with pressure. The density, on the other hand, is affected. However, as it is already 3 orders of magnitude smaller than that of the droplet, further decreases with the pressure seem unlikely to have a significant effect. This work addresses the modelling of the air pressure in the droplet collision problem. We believe that progress made here will provide useful clues for solving the more difficult problem of drop impact.

One of the most difficult issues in multiphase flows is modelling the topological change of interfaces. There are two basic types of topology change. The easier one is filamentary break-up, where surface tension is the driv-

\footnotetext{
a)Email: j1305@cam.ac.uk
}

ing mechanism and universal macroscopic solutions based on continuum mechanics have been found ${ }^{21}$. In the second type, interface merging, continuum mechanics is not sufficient to describe the physical process. The final stage of the interfacial dynamics involves molecular Van der Waals forces (prevalent on scales of 10-40 nm) and thereby also rarefied gas flow (the mean free path of air is of order of $100 \mathrm{~nm}$ at $1 \mathrm{~atm}$ ). A successful model must incorporate these microscopic physics in order to accurately predict the interface merging from first principles. Droplet collision represents a canonical example of interface merging.

We consider incompressible flows of two different phases with density $\rho_{i}$ and viscosity $\mu_{i}$, where $i=$ liquid or gas. The surface tension coefficient is denoted as $\sigma$. The flows of the phases are governed by the incompressible Navier-Stokes equations ${ }^{22}$. Given that they are assumed axisymmetric, the continuity equation reads

$$
\frac{1}{r} \frac{\partial}{\partial r}(r u)+\frac{\partial v}{\partial z}=0
$$

and the momentum equations are

$$
\begin{gathered}
\rho \frac{d u}{d t}=-\frac{\partial p}{\partial r}+\frac{1}{r} \frac{\partial}{\partial r}\left(r \tau_{r r}\right)+\frac{\partial}{\partial z}\left(\tau_{r z}\right)-\frac{2 \mu u}{r^{2}}, \\
\rho \frac{d v}{d t}=-\frac{\partial p}{\partial z}+\frac{1}{r} \frac{\partial}{\partial r}\left(r \tau_{z r}\right)+\frac{\partial}{\partial z}\left(\tau_{z z}\right),
\end{gathered}
$$

where the notation $\frac{d}{d t}$ denotes the material derivative $\frac{\partial}{\partial t}+u \frac{\partial}{\partial r}+v \frac{\partial}{\partial z}, p$ is the pressure, $u$ and $v$ the radial and axial velocities, $\tau_{r r}, \tau_{r z}, \tau_{z r}$ and $\tau_{z z}$ the four components of the stress tensor $\boldsymbol{\tau}$. The boundary condition on the interface, expressing the force balance between the surface 


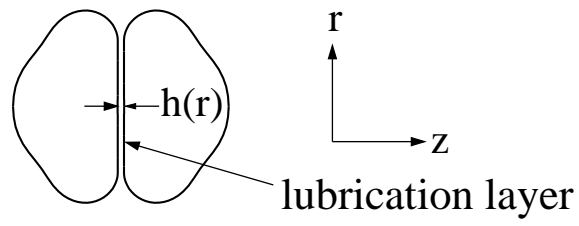

FIG. 1. Configuration of two head-on colliding droplets of same size with the formation of a thin flat lubrication layer.

tension, stress and Van der Waals forces, is:

$$
[(-p \mathbf{I}+\boldsymbol{\tau}) \cdot \mathbf{n}]_{-}^{+}=\left(\sigma \kappa-\frac{A_{H}}{6 \pi h^{3}(\mathbf{x})}\right) \mathbf{n},
$$

where $[\cdot]_{-}^{+}$is the difference between the enclosed quantity on the two sides of the interface, $\kappa$ its local curvature, and $\mathbf{n}$ its local unit normal, $A_{H}$ the Hamaker constant and $h(\mathbf{x})$ the minimum distance from point $\mathbf{x}$ to the other interface. Here the Van der Waals forces are attractive forces and are approximated by the disjoint pressure model ${ }^{23,24}$. These forces, which are inversely proportional to the cubic power $h^{3}$, only become effective when $h$ is order of $10 \mathrm{~nm}$.

The dynamics of the collision are dominated by the slow drainage of a flat interdroplet gas film of thickness $h(r)$ with $d h / d r \ll 1$ (see Fig. 1 ). When the film thickness reaches the order as the mean free path, the continuum assumption fails; the film flow is now governed by the Boltzmann equation, instead of the Navier-Stokes equations. A hybrid method coupling the Navier-Stokes and Boltzmann equations is desirable but complicated.

Our aim is to develop a macroscopic model solely based on the Navier-Stokes equation. Zhang and Law ${ }^{12}$ have developed the first comprehensive theory for droplet collisions based on 4 ordinary differential equations. They showed that the pressure within the lubrication layer can be expressed in a unified form for the continuum and rarefied flows as

$$
p_{g}=\frac{3 \mu_{g}}{\Delta h^{3}}\left(r^{2}-a^{2}\right)\left(\frac{\mathrm{d} h}{\mathrm{~d} t}+2 \kappa h\right),
$$

where the correction factor $\Delta$ is a function of the Knudsen number $\mathrm{Kn}=\lambda / h$ (with $\lambda$ the mean free path). See Eqn. (73) in ref 13 for an explicit formula for $\Delta$.

This result is used in the present work based on the following observations: (1) the Boltzmann equation is only needed in the lubrication layer; (2) the continuum flow solution based on lubrication theory is an accurate approximation of the Navier-Stokes equations; (3) the rarefied flow solution in the lubrication layer only differs from the continuum flow one by a correction factor. The crucial step in this work is the realization that the lubrication resistance force can be reproduced by solving the Navier-Stokes using a variable (effective) viscosity such that in the lubrication layer $\mu=\mu_{\mathrm{g}} / \Delta(\mathrm{Kn})$. The effective viscosity is about 1 order of magnitude smaller than the macroscopic viscosity $\mu_{g}$ at $\mathrm{Kn}=1$ and decreases as $h^{1.1551}$ with the film thickness for $\mathrm{Kn} \geq 1$; the effect of rarefied gas dynamics is to reduce the lubrication resistance force and therefore to encourage the droplet coalescence.

This model has the elegant property that the continuum and rarefied flows are unified in a single set of Navier-Stokes equations with a variable (effective) viscosity, and the transition between the two flow regimes is effected smoothly via the correctional factor $\Delta(\mathrm{Kn})$ within the lubrication layer. Unlike Zhang and Law ${ }^{12}$, whose droplet-collision theory includes an empirical coefficient that is not universal, we use no adjustable parameters.

The droplet collision involves length scales across more than 5 orders of magnitude. It is not yet feasible to capture all these with a fixed-grid method. We have instead developed a state of the art moving adaptive mesh method. The idea of using moving mesh methods for computing multiphase flows is an old one, dated back to the $1980 \mathrm{~s}^{25}$. We summarize here the distinct features of our method : (1) our mesh moves with the interfaces between different phases so that the interfaces are always lines of the mesh; (2) in our mesh each triangle contains only one phase, no matter how thin the lubrication layer is. A direct consequence is that an accurate representation of the interfacial boundary conditions is obtained; (3) the Navier-Stokes equations are resolved in both phases simultaneously using a Finite Element Method: second degree polynomials are used to approximate the velocity in each triangle. In the lubrication theory, the velocity profile in the air film is parabolic. Therefore, our numerical method will capture the exact solution in the lubrication layer, even with only a single layer of elements. The reader is referred to the Supplemental Material [26] and refs [27-34] for the details.

Real material properties, listed in Table I, are used in this study. The air properties in Table $\mathrm{I}$ are for $1 \mathrm{~atm}$ pressure. The mean free path is inversely proportional to the density. Under constant temperature, the air density is proportional to the ambient pressure while its mean free path is inversely proportional. The effect of the air pressure on the viscosity and the surface tension is negligible. The relevant dimensionless numbers are

$$
\begin{gathered}
W e=\frac{\rho_{\mathrm{l}} D U^{2}}{\sigma}, C a=\frac{\mu_{\mathrm{g}} U}{\sigma}, \bar{\lambda}=\frac{\lambda}{D}, \\
\bar{A}_{H}=\frac{A_{H}}{6 \pi \rho_{\mathrm{l}} U^{2} D^{3}}, \bar{\rho}=\frac{\rho_{g}}{\rho_{l}}, \bar{\mu}=\frac{\mu_{l}}{\mu_{g}} .
\end{gathered}
$$

where $U$ is the droplet relative speed and $D$ the diameter. On increasing the viscosity ratio $\bar{\mu}_{l}$, the droplets' deformation (hence the lubrication resistance) will be reduced, and the coalescence enhanced. Increasing $\bar{A}_{H}$ increases the van der Waals attractive force and enhances the coalescence too. It will be shown that the density ratio $\bar{\rho}$ is unimportant in the limit $\rho_{g} \ll \rho_{l}$.

It is well known that the outcome of a binary droplet collision is nonmonotonic as the collision intensity increases; for small $W e$ (soft collision), there is a transition 
TABLE I. Physical properties of the liquids and gas in the simulation

\begin{tabular}{cc}
\hline \hline air density & $1.225 \mathrm{~kg} \mathrm{~m}^{-3}$ \\
air viscosity & $1.827 \times 10^{-5} \mathrm{~N} \mathrm{~s} \mathrm{~m}^{-2}$ \\
mean free path & $6.9 \times 10^{-8} \mathrm{~m}$ \\
tetradecane density & $762.0 \mathrm{~kg} \mathrm{~m}^{-3}$ \\
tetradecane viscosity & $2.128 \times 10^{-3} \mathrm{~N} \mathrm{~s} \mathrm{~m}^{-2}$ \\
tetradecane-air surface tension & $2.65 \times 10^{-2} \mathrm{~N} \mathrm{~m}^{-1}$ \\
tetradecane Hamaker constant & $5.0 \times 10^{-20} \mathrm{~J}$ \\
water density & $1000.0 \mathrm{~kg} \mathrm{~m}^{-3}$ \\
water viscosity & $1.00 \times 10^{-3} \mathrm{~N} \mathrm{~s} \mathrm{~m}^{-2}$ \\
water-air surface tension & $7.3 \times 10^{-2} \mathrm{~N} \mathrm{~m}^{-1}$ \\
water Hamaker constant & $3.7 \times 10^{-20} \mathrm{~J}$ \\
\hline \hline
\end{tabular}

from coalescence to bouncing at $W e_{S}$, while one from bouncing to coalescence (at $W e_{H}$ ) is found for large $W e$ (hard collision). Boundaries between the various regimes are significantly influenced by the ambient gas pressure ${ }^{19}$. We have investigated the collision regimes for tetradecane and water droplets. Two radii, $R=107.2 \mu \mathrm{m}$ and $167.6 \mu \mathrm{m}$, were considered for tetradecane. The experimental radius for water droplets varies between $100 \mu \mathrm{m}$ and $300 \mu \mathrm{m}$; only $R=150 \mu \mathrm{m}$ was considered in this study. Our predicted critical Weber numbers together with the corresponding experimental values ${ }^{12,19}$ are summarized in Table II, and show remarkably good agreement.

Fig. 2 shows the collision sequence of the computed tetradecane droplets for Weber number 9.33 at 1 atm. The computed interface positions are superposed on the experimental results ${ }^{32}$ to demonstrate the close correspondence between the two.

A striking experimental observation is that the bouncing regime disappears for tetradecane droplets when the ambient pressure is reduced to $0.6 \mathrm{~atm}$. This is confirmed by our numerical simulations. Fig. 3 plots the minimum gap thickness $h_{\min }$ as a function of time $t$ for $W e=9.33$ and radius $R=167.6 \mu \mathrm{m}$ (the length and time in this figure and Fig. 4 are made dimensionless with characteristic length and velocity $D$ and $U$ ). The thick solid line represents the data at $1 \mathrm{~atm}$ and the broken line that at $0.6 \mathrm{~atm}$. For the latter case, the gas density is decreased by a factor 0.6 and the mean free path increased by $5 / 3$.

According to the lubrication theory, formula (5), the air density plays no role at all in the lubrication force. The gas density is already 3 orders of magnitude smaller than the liquid, so while a change of $40 \%$ may have a minor influence at the early stage of rapid droplet approach, it surely does not significantly affect the more relevant slow drainage stage, and hence the outcome of the collision. To support our assertion, we plot also in Fig. 3 the minimum gap thickness for the case when only the density is reduced (represented by $\bullet$ ), and for the case when only the mean free path is increased (represented by $\circ$ ). The data for the former case follows closely the

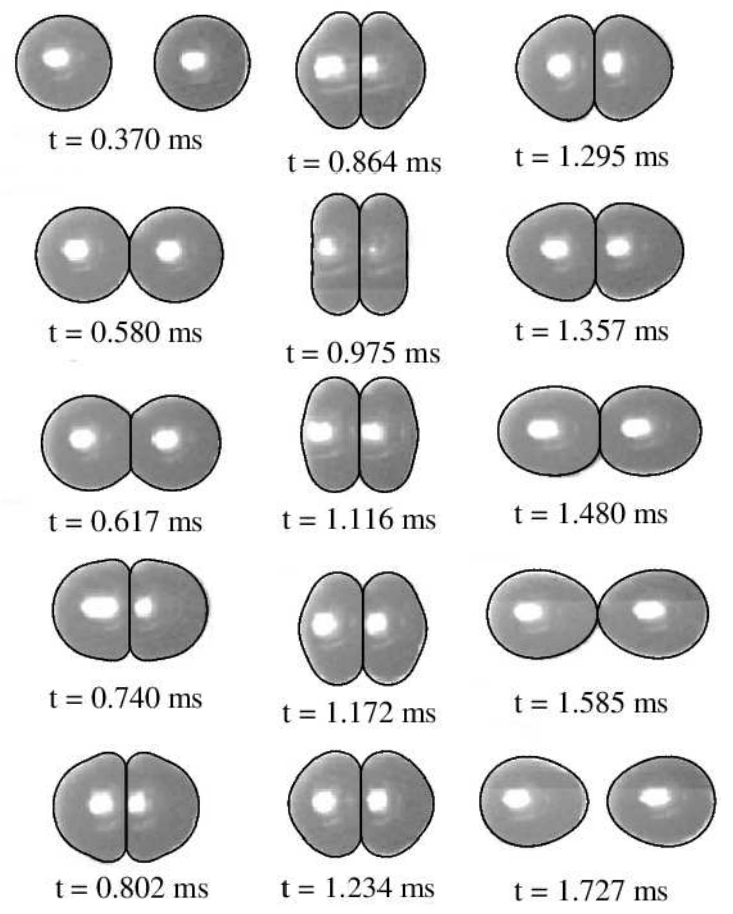

FIG. 2. Bouncing collision sequence of tetradecane droplets in $1 \mathrm{~atm}$ air. $R=167.6 \mu \mathrm{m}$, and $W e=9.33$. Comparison between the experimental results of Ref. 32 and the results from the computation (present study) represented by thick solid lines.

1 atm curve, while that for the latter matches the 0.6 atm curve. We conclude that the influence of the atmospheric pressure is via the increase of the mean free path (the rarefied gas effect). We note that a similar influence of air via the mean free path has been suggested by Riboux et $\mathrm{al}^{35}$ for the splashing problem, and by Marchand et $\mathrm{al}^{36}$ for air entrainment by moving contact lines.

No droplet size effect has previously been reported. Our calculation reveals that at $1 \mathrm{~atm}$, the bouncing region is much smaller for tetradecane droplets of $R=$ $107.2 \mu \mathrm{m}$ than those of $R=167.6 \mu \mathrm{m}$. On the other hand, this difference is much smaller at high ambient pressure $(2.4 \mathrm{~atm})$. The experimental soft transition $W e_{S}=2.3$ was reported for $R=107.2 \mu \mathrm{m}$ while the hard transition $W e_{H}=12.3$ for $R=167.6 \mu \mathrm{m}$ (see ref. 32). Our numerical $W e_{S}$ (between 2.4 and 2.5) for $R=107.2 \mu \mathrm{m}$ is very close to the experimental value reported for the same radius. For $R=167.6 \mu \mathrm{m}$, our numerical $W e_{H}$ (between 14.9 and 15) is also a good approximation of the experimental one (12.3).

To shed light on this observation, we consider the opposing effects of $C a=\mu_{g} \sqrt{W e} / \sqrt{\rho \sigma D}$ and $\bar{\lambda}=\lambda / D$. For the same liquid and Weber number, the larger $D$ is , the smaller is $C a$, the smaller the droplet deformation and the higher the chance of coalescence. On the other hand, the larger $D$ is, the smaller is $\bar{\lambda}$, the less the rarefied gas effect, and the less the chance of coalescence. To make our argument quantitative, we plot in 
TABLE II. Comparison of the predicted and experimental transition $W e$ for the collision of tetradecane (two radii) and water droplets in air.

\begin{tabular}{|c|c|c|c|c|c|}
\hline \multirow[b]{3}{*}{$W e_{S}(\exp )$} & \multicolumn{2}{|c|}{ Tetradecane $(1.0 \mathrm{~atm})$} & \multirow[t]{2}{*}{ Tetradecane $(0.6 \mathrm{~atm})$} & \multicolumn{2}{|c|}{ Tetradecane $(2.4 \mathrm{~atm})$} \\
\hline & $R=107.2 \mu \mathrm{m}$ & $R=167.6 \mu \mathrm{m}$ & & $R=107.2 \mu \mathrm{m}$ & $R=167.6 \mu \mathrm{m}$ \\
\hline & 2.3 & Not available & Coalescence only & Not available & Not available \\
\hline$W e_{S}$ (present) & $2.3-2.4$ & $1.1-1.2$ & Coalescence only & $0.4-0.5$ & $0.5-0.6$ \\
\hline$W e_{H}(\exp )$ & Not available & 12.3 & Coalescence only & 30.0 & 30.0 \\
\hline$W e_{H}$ (present) & $6.2-6.3$ & $14.9-15.0$ & Coalescence only & $18.5-19.0$ & $19.3-19.5$ \\
\hline & \multicolumn{2}{|c|}{ Water $(1.0$ atm $)$} & Water (2.7 atm) & \multicolumn{2}{|c|}{ Water $(8.0$ atm $)$} \\
\hline$W e_{S}(\exp )$ & \multicolumn{2}{|c|}{ Coalescence only } & 3.0 & \multicolumn{2}{|c|}{ Not available } \\
\hline$W e_{S}$ (present) & \multicolumn{2}{|c|}{ Coalescence only } & $3.7-3.8$ & \multicolumn{2}{|c|}{$3.5-3.6$} \\
\hline$W e_{H}(\exp )$ & \multicolumn{2}{|c|}{ Coalescence only } & 6.0 & \multicolumn{2}{|c|}{13.0} \\
\hline$W e_{H}$ (present) & \multicolumn{2}{|c|}{ Coalescence only } & $6.5-6.6$ & \multicolumn{2}{|c|}{$13.0-13.5$} \\
\hline
\end{tabular}

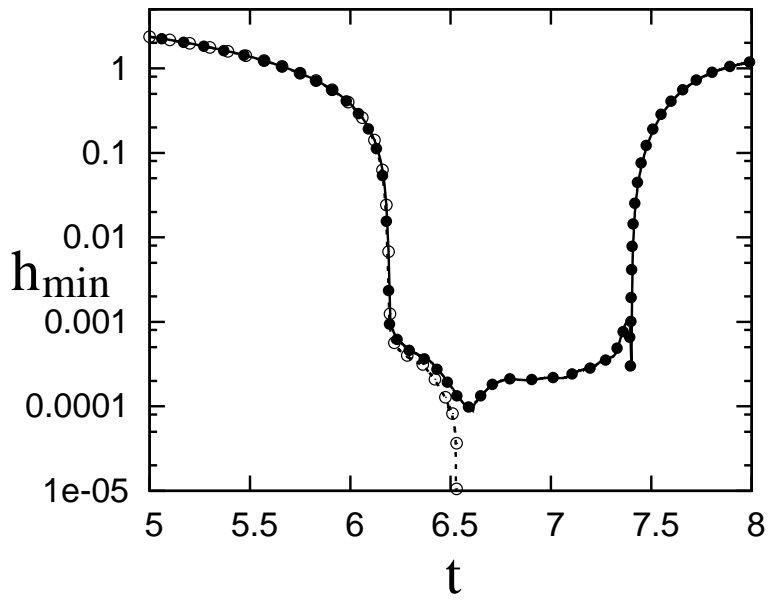

FIG. 3. The minimum gap thickness $h_{\min }$ as a function of time $t$ (dimensionless quantities calculated with droplet diamter and speed) for $W e=9.33$. - conditions as Fig. 2; - -, pressure reduced to $0.6 \mathrm{~atm} ; \bullet \bullet$, density reduced, mean free path unchanged; $\circ$, mean free path increased, density unchanged.

Fig. 4 the minimum gap thickness as a function of time for the two radii at $1 \mathrm{~atm}$ and $2.4 \mathrm{~atm}$ all with $W e=2.5$. For the larger $D$, we observe that the minimum thickness is slightly smaller at the initial stage. However, by and large, at $1 \mathrm{~atm}$, the determining factor is the dimensionless mean free path; at the lowest values, the large droplet data $\left(\bullet, \bar{\lambda}=2.06 \times 10^{-4}\right)$ is significantly higher. This is because the reduction of the viscosity due to the rarefied gas effect is much larger for the small droplets $\left(\bar{\lambda}=3.22 \times 10^{-4}\right)$. Finally, when the ambient pressure is increased to $2.4 \mathrm{~atm}$, both mean free paths are reduced 2.4 times (bringing them closer to the effective range of Van der Waals forces), the rarefied gas effect is less important, and the difference is hence smaller.

This work addresses one important question: the modelling of the effect of the air pressure in the droplet col-

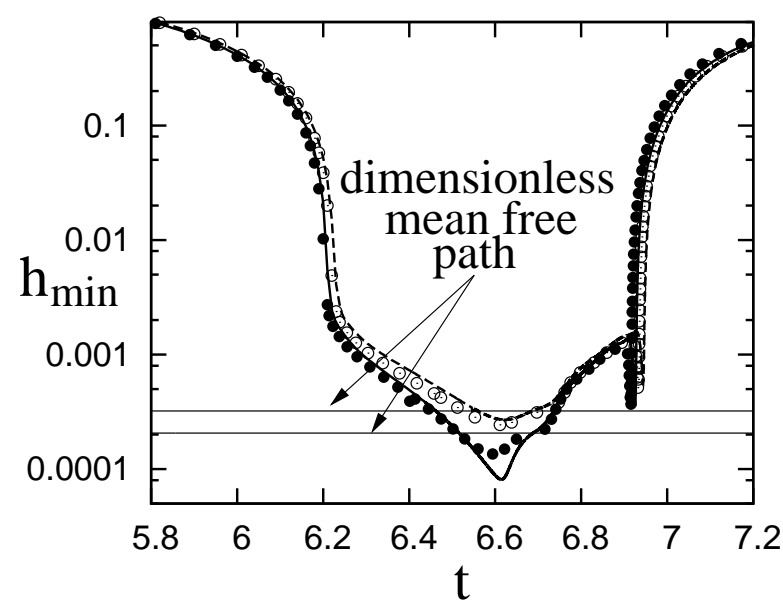

FIG. 4. The minimum gap thickness $h_{\min }$ as a function of time $t$ (dimensionless quantities) for $W e=2.5$. - tetradecane droplets of radius $107.2 \mu \mathrm{m}$ at $1 \mathrm{~atm}$; - - ambient pressure increased to $2.4 \mathrm{~atm}$; $\bullet$, radius $167.6 \mu \mathrm{m}$ at $1 \mathrm{~atm}$; $\circ$, radius $167.6 \mu \mathrm{m}$ at $2.4 \mathrm{~atm}$. The two horizontal lines show values of the dimensionless mean free paths $\bar{\lambda}=\lambda / D$ at 1 atm for the two radii $\left(3.22 \times 10^{-4}\right.$ and $2.06 \times 10^{-4}$ respectively $)$.

lision problem. A theoretical breakthrough was made by Gopinath et al. through the introduction of compressibility and non-continuum effects ${ }^{37,38}$. The Van der Waals forces and large deformation of droplet were included in Zhang and Law's model ${ }^{12}$. The present work is the first work which has successfully incorporated both microscopic Van der Waals forces and rarefied gas effects into the macroscopic Navier-Stokes equations. Our theory is based entirely on fundamental laws of physics. It contains no adjustable (empirical) parameters for data fitting, and makes no assumption on the droplet shape; the interface location is part of the solution that we seek. The numerical implementation handles the huge ( 5 orders of magnitude) length scale range of the problem via a unique state of the art moving adaptive mesh method ${ }^{33}$. The results compare very well with experi- 
mental data ${ }^{12,32}$. Given the extreme sensitivity of the rupture of the interdroplet gas film, the numerical boundaries of soft coalescence-bouncing and hard bouncingcoalescence regimes are remarkably close to the experimental ones. Our investigations have also led to new, sometimes counter-intuitive, insights regarding the film rupture. We found that the effect of the gas density is minor, and the ambient pressure and the droplet size determine the collision outcome through the rarefied gas effect, which reduces the effective gas viscosity (via its dependence on the mean free path). Future work will attempt to incorporate additional physics, for instance, the effect of the liquid vapor, into our theory, and to use it to elucidate the suppression of the drop splashing, a critical outstanding problem in the multiphase flows in the decade.

\section{ACKNOWLEDGMENTS}

We thank Dr. Shengyong Xia and Dr. James Sprittles for stimulating discussions and Dr. Will Graham for proofreading the manuscript. Part of this work was conducted at Beijing Computational Science Research Center. We thank Prof. Shili Luo for his hospitality.

${ }^{1}$ A. Yarin, "Drop impact dynamics: Splashing, spreading, receding, bouncing," Annual review of fluid mechanicS 38, 159-192 (2006).

${ }^{2}$ S. Mandre, M. Mani, and M. Brenner, "Precursors to splashing of liquid droplets on a solid surface," Phys. Review Letters 102(13), 134502 (2009).

${ }^{3}$ J. Eggers, M. Fontelos, C. Josserand, and S. Zaleski, "Drop dynamics after impact on a solid wall: Theory and simulations," Phys. Fluids 20 (6), 062101 (2010).

${ }^{4}$ L. Duchemin and C. Josserand, "Curvature singularity and filmskating during drop impact," Phys. Fluids 23(9), 091701 (2011).

${ }^{5} \mathrm{~J}$. Adam, N. Lindblad, and C. Hendrick, "Collision coalescence and disruption of water droplets," J. Applied Phys. 39(11), 5173 (1968).

${ }^{6}$ S. Bradley and C. Stow, "Collisions between liquid-drops," Philos. Trans. R. Soc. Ser. A 287, 635 (1978).

${ }^{7} \mathrm{~N}$. Ashgriz and Y. Poo, "Coalescence and separation in binary collisions of liquid drops," J. Fluid. Mech. 221, 183-204 (1990).

${ }^{8}$ Y. Jiang, A. Umemura, and C. Law, "An experimental investigation on the collision behaviour of hydrocarbon droplets," J. Fluid Mech. 234, 171 (1992).

${ }^{9}$ G. Bach, D. Koch, and A. Gopinath, "Coalescence and bouncing of small aerosol droplets," J. Fluid Mech. 518, 157-185 (2004).

${ }^{10}$ K. Yokoi, D. Vadillo, J. Hinch, and I. Hutchings, "Numerical studies of the influence of the dynamic contact angle on a droplet impacting on a dry surface," Phys. of Fluids 21, 072102 (2009).

${ }^{11} \mathrm{~S}$. Mandre, M. Mani, and M. Brenner, "Events before droplet splashing on a solid surface," J. Fluid Mech. 647, 163-185 (2010).

${ }^{12} \mathrm{P}$. Zhang and C. Law, "An analysis of head-on droplet collision with large deformation in gaseous medium," Phys. Fluids 23, 042102 (2011).

${ }^{13} \mathrm{~J}$. Sprittles and Y. Shikhmurzaev, "The dynamics of liquid drops and their interaction with solids of varying wettabilities," Phys. Fluids 24, 082001 (2012).

${ }^{14}$ J. Kolinski, S. Rubinstein, S. Mandre, M. Brenner, D. Weitz, and L. Mahadevan, "Skating on a film of air: Drops impacting on a surface," Phys. Review Letters 108(7), 074503 (2012).

${ }^{15}$ L. Duchemin and C. Josserand, "Rarefied gas correction for the bubble entrapment singularity in drop impacts," Comptes Rendus Mecanique 340 (11-12), 797-803 (2012).

${ }^{16}$ J. Kolinski, L. Mahadevan, and S. Rubinstein, "Lift-off instability during the impact of a drop on a solid surface," Phys. Review Letters 112(13) (2014).

${ }^{17}$ D. Lycett-Brown, K. Luor, R. Liu, and P. Lv, "Binary droplet collision simulations by a multiphase cascaded lattice boltzmann method," Phys. Fluids 26, 023303 (2014).

${ }^{18} \mathrm{~S}$. Xia and C. Hu, "Numerical investigation of head-on binary collision of alumina droplets," Journal of Propulsion and Power 31, 416-428 (2014).

${ }^{19} \mathrm{~J}$. Qian and L. CK, "Regimes of coalescence and separation in droplet collision," J. Fluid Mech. 331, 59-80 (1997).

${ }^{20} \mathrm{~L}$. Xu, W. Zhang, and R. Nagel, S., "Drop splashing on a dry smooth surface," Phys. Review Letters 94, 184505 (2005).

${ }^{21} \mathrm{~J}$. Eggers, "Nonlinear dynamics and breakup of free-surface flows," Reviews of modern physics 69, 865 (1997).

${ }^{22} \mathrm{~J}$. Ferziger and M. Peric, Computational methods for fluid dynamics (Springer, 1996).

${ }^{23} \mathrm{~W}$. Zhang and J. Lister, "Similarity solutions for van der waals rupture of a thin film on a solid substrate," Phys. Fluids 11, 2454-2462 (1999).

${ }^{24}$ K. Savettaseranee, T. Papageorgiou, D., P. Petropoulos, and S. Tilley, B., "The effect of electric fields on the rupture of thin viscous films by van der waals forces," Phys. Fluids 15(3), 641$652(2003)$.

${ }^{25}$ E. Fyfe, D., S. Oran, E., and M. Fritts, "Surface-tension and viscosity with lagrangian hydrodynamics on a triangular mesh," J. Comput. Phys. 76(2), 349-384 (1988).

26 "See the supplemental material at [url], which includes refs. [2734]."

${ }^{27} \mathrm{~J}$. Li, "Calcul d'Interface Affine par Morceaux (Piecewise Linear Interface Calculation)," C. R. Acad. Sci. Paris t.320 série IIb, 391-396 (1995)

${ }^{28}$ D. Gueyffier, J. Li, A. Nadim, R. Scardovell, and S. Zaleski, "Volume-of-fluid interface tracking and smoothed surface stress methods applied to multiphase flow and pendant drop pinching," J. Comput. Phys. 152, 423-456 (1999).

${ }^{29} \mathrm{~J}$. Li, Y. Renardy, and M. Renardy, "Numerical simulation of breakup of a viscous drop in simple shear flow through a volumeof-fluid method," Phys. Fluids 12(2), 269-282 (2000).

${ }^{30} \mathrm{~J}$. Li, M. Hesse, J. Ziegler, and A. M. Woods, "An arbitrary Lagrangian Eulerian method for moving-boundary problems and its application to jumping over water," J. Comput. Phys. 208, 289-314 (2005).

${ }^{31}$ J. Etienne, J. Hinch, E., and J. Li, "A Lagrangian-Eulerian approach for the numerical simulation of free-surface flow of a viscoelastic material," J. Non-Newtonian Fluid Mech. 136, 157166 (2006).

${ }^{32}$ K. Pan, C. Law, and B. Zhou, "Experimental and mechanistic description of merging and bouncing in head-on binary droplet collision," J. Applied Phys. 103, 064901 (2008).

${ }^{33} \mathrm{~J} . \mathrm{Li}$, "An arbitrary Lagrangian Eulerian method for three-phase flows with triple junction points," J. Comput. Phys. 251, 1-16 (2013).

${ }^{34}$ M. Kwakkel, W.-P. Breugem, and B. Boersma, "Extension of a CLSVOF method for droplet-laden flows with a coalescence/breakup model," J. Comput. Phys. 253, 166-188 (2013).

${ }^{35} \mathrm{G}$. Riboux and J. Gordillo, "Experiments of drops impacting a smooth solid surface: A model of the critical impact speed for drop splashing," Physical review letters 113, 024507 (2014).

${ }^{36}$ A. Marchand, T. Chan, J. Snoeijer, and B. Andreotti, "Air entrainment by contact lines of a solid plate plunged into a viscous fluid," Physical review letters 108, 204501 (2012).

${ }^{37}$ A. Gopinath, B. Chen, S., and L. Koch, D., "Lubrication flows between spherical particles colliding in a compressible noncontinuum gas," J. Fluid Mech. 344, 245-269 (1997).

${ }^{38} \mathrm{~S}$. Mandre and P. Brenner, M., "The mechanism of a splash on a dry solid surface," J. Fluid Mech. 690, 148-172 (2012). 